\section{POS1224 RHEUMATIC MANIFESTATIONS IN COVID-19 PATIENTS - SINGLE-CENTER EXPERIENCE AMIDST THE PANDEMIC}

S. Bogdanova-Petrova ${ }^{1,2}$, T. Georgiev $^{1,2}$, G. Gerganov $^{2,3}$, S. Hristova ${ }^{2,4}$, S. Dimitrov ${ }^{1,2}$, T. Shivacheva ${ }^{1,2} .{ }^{1}$ Medical University - Varna, First Department of Internal Medicine, Varna, Bulgaria; ${ }^{2}$ St. Marina's Hospital, Rheumatology Clinic, Varna, Bulgaria; ${ }^{3}$ Medical University - Varna, Department of Propedeutics of Internal Medicine, Varna, Bulgaria; ${ }^{4}$ Medical University - Varna, Department of General Medicine and Clinical Laboratory, Varna, Bulgaria

Background: Recently evolved from a monochromic flu-like disease to a polysyndromic "spectrum of disease," our understanding of coronavirus disease 2019 (COVID-19) is still far from being complete [1]. Hyperinflammation involving not only the lungs but also the musculoskeletal system, skin, cardiovascular, genitourinary systems is immune-mediated resembling the flares of a full-blown rheumatic disease [2,3].

Objectives: To describe the prevalence and type of rheumatic manifestations in a cohort of COVID-19 patients hospitalized in the COVID-19 rheumatology department in University Hospital St. Marina, Varna, Bulgaria.

Methods: In the present single-center cohort study, a retrospective database analysis was performed among all COVID-19 patients hospitalized from 1 Dec 2020 to 22 Jan 2021. All 243 patients (age 19 - 93 years) were treated for moderate or severe SARS-CoV-2 infection confirmed by laboratory tests, including positive polymerase chain reaction (PCR) test, and imaging modality. Inpatient treatment included antibiotics, dexamethasone, anticoagulants, and antiviral drug remdesevir (optional). Detailed disease history and clinical examination were carried out by a fully certified rheumatologist and/or specialist in internal medicine.

Results: Among all 243 COVID-19 patients, those with prominent self-reported myalgia and arthralgia were $26 \%(n=63)$ and $21.3(n=52)$, respectively. We had $4(1.6 \%)$ cases of newly developed cutaneous vasculitis and $2(0.8 \%)$ cases of severe Raynaud's phenomenon after SARS-CoV-2 infection onset. Two patients experienced severe muscle weakness, had elevated creatine phosphokinase, and were diagnosed with inflammatory myopathy secondary to COVID-19. Lupus-like syndrome was observed in $2(0.8 \%)$ patients.

Conclusion: Rheumatic manifestations are part of the heterogeneous spectrum of COVID-19 disease. Amidst the COVID-19 crisis, each newly onset rheumatic manifestation warrants exclusion of SARS-CoV-2 infection. Therefore, a rheumatologist should be a part of a multidisciplinary approach towards the COVID-19 treatment.

REFERENCES:

[1] Misra DP, Agarwal V, Gasparyan AY, Zimba O. Rheumatologists' perspective on coronavirus disease 19 (COVID-19) and potential therapeutic targets. Clin Rheumatol. 2020;39(7):2055-2062

[2] Georgiev T, Angelov AK. Complexities of diagnosis and management of COVID-19 in autoimmune diseases: Potential benefits and detriments of immunosuppression. World J Clin Cases. 2020;8(17):3669-3678

[3] Ciaffi J, Meliconi R, Ruscitti P, Berardicurti O, Giacomelli R, Ursini F. Rheumatic manifestations of COVID-19: a systematic review and meta-analysis. BMC rheumatology. 2020 Dec;4(1):1-5.

Disclosure of Interests: None declared

DOI: 10.1136/annrheumdis-2021-eular.2836

\section{POS1225 \\ INFLUENCE OF COLCHICINE PRESCRIPTION IN COVID19-RELATED HOSPITAL ADMISSIONS: A SURVIVAL ANALYSIS}

M. P. Álvarez ${ }^{1}$, A. Madrid García ${ }^{1,2}$, I. Perez-Sancristobal ${ }^{1}$, J. I. Colomer ${ }^{1}$, L. León ${ }^{1}$, J. A. Jover', B. Fernandez ${ }^{1}$, L. Abasolo', L. Rodriguez Rodriguez'1 ${ }^{1}$ Health Research Institute (IdISSC), Hospital Clínico San Carlos,

Rheumatology Department, Madrid, Spain; ${ }^{2}$ Escuela Técnica Superior de Ingenieros de Telecomunicación (ETSIT), Universidad Politécnica de Madrid, Madrid, Spain

Background: Severe acute respiratory syndrome coronavirus 2 (SARS-CoV-2), triggers the innate immune system, leading in severe cases, an excessive immune response, which can lead to high levels of pro-inflammatory cytokines promoting a "cytokine storm".

To modulate this exaggerated inflammatory response, several clinical trials with already approved and well-known therapeutic agents that inhibit the inflammatory response, are being carried out. However, none of these drugs seems to achieve the desired results when treating COVID19.

Colchicine, a drug often used in the management of patients with Rheumatic and Musculoskeletal diseases (RMDs), is one of the several drugs that are being currently tested for efficacy in COVID19 due to its anti-inflammatory effects.
Objectives: To analyze association between colchicine prescription and COVID19-related hospital admissions in patients with Rheumatic and Musculoskeletal diseases (RMDs).

Methods: Patients attending a rheumatology outpatient clinic from a tertiary care center in Madrid, Spain, from $1^{\text {st }}$ September 2019 to $29^{\text {th }}$ February 2020 were included.

Patients were assigned as exposed or unexposed based on whether they were prescribed with colchicine in their last visit to the clinic during the 6 months before the start of the observation period. Treatment changes during the observation period were also considered. The primary outcome was COVID19-related hospital admissions occurring between March $1^{\text {st }}$ and May $20^{\text {th }}, 2020$. Secondary outcome included COVID19-related mortality. Several weighting techniques for data balancing, based and non-based on the propensity score, followed by Cox regressions were performed to estimate the association of colchicine prescription on both outcomes.

Results: 9,379 patients entered in the study, with 406 and 9,002 exposed and unexposed follow-up periods, respectively. Generalized Boosted Models (GBM) and Empirical Balancing Calibration Weighting (EBCW) methods showed the best balance for COVID19-related hospital admissions. Colchicine prescription did not show a statistically significant association after covariable balancing ( $p$-value $=$ 0.195 and 0.059 for GBM and EBCW, respectively). Regarding mortality, the low number of events prevented a success variable balancing and analysis.

Conclusion: Colchicine prescription does not play a significant protective or risk role in RMD patients regarding COVID19-related hospital admissions. Our observations could support the maintenance of colchicine prescription in those patients already being treated, as it is not associated with a worse prognosis.

REFERENCES:

[1] Fernandez-Gutierrez B. COVID-19 with Pulmonary Involvement. An Autoimmune Disease of Known Cause. Reumatol Clin 2020; 16: 253-254.

[2] Coperchini F, Chiovato L, Croce L, et al. The cytokine storm in COVID-19: An overview of the involvement of the chemokine/chemokine-receptor system. Cytokine Growth Factor Rev 2020; 53: 25-32.

[3] Shaffer L. 15 drugs being tested to treat COVID-19 and how they would work. Nat Med. Epub ahead of print 15 May 2020. DOI: 10.1038/ d41591-020-00019-9.

[4] Fernandez-Gutierrez B, Leon L, Madrid A, et al. Hospital admissions in inflammatory rheumatic diseases during the COVID-19 pandemic: incidence and role of disease modifying agents. medRxiv 2020; 2020.05.21.20108696.

[5] Freites Nuñez DD, Leon L, Mucientes A, et al. Risk factors for hospital admissions related to COVID-19 in patients with autoimmune inflammatory rheumatic diseases. Ann Rheum Dis 2020; 1-7.

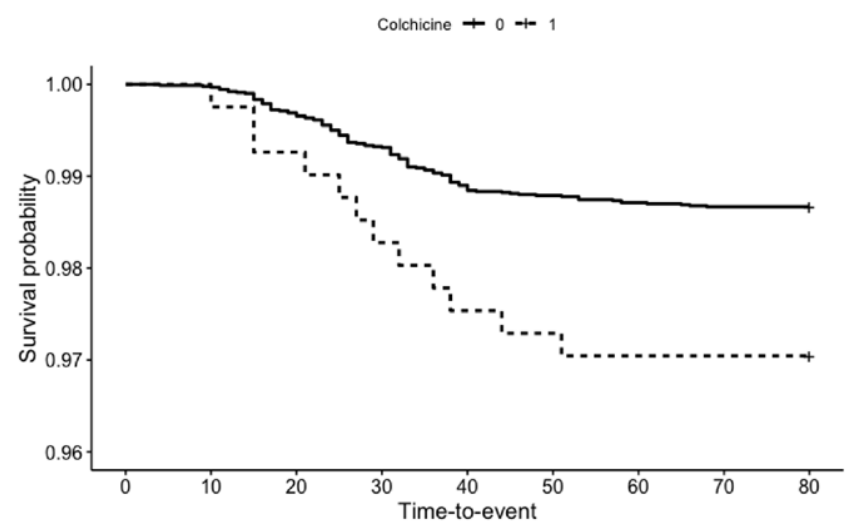

Disclosure of Interests: None declared

DOI: 10.1136/annrheumdis-2021-eular.2845

\begin{tabular}{|l|l|}
\hline POS1226 & THE COURSE OF CORONAVIRUS DISEASE \\
& 2019 (COVID-19) IN PATIENTS WITH SJOGREN'S \\
SYNDROME TREATED WITH ANTI-CD20 \\
MONOCLONAL ANTIBODY (RITUXIMAB)
\end{tabular}

E. Sokol ${ }^{1}$, A. Torgashina ${ }^{1}$, B. Chalcev ${ }^{1}$, J. Khvan ${ }^{1}$, O. Golovina ${ }^{1} .{ }^{1}$ V.A. Nasonova Research Institute of Rheumatology, Laboratory of Intensive Care of Rheumatic Diseases, Moscow, Russian Federation

Background: The severe acute respiratory syndrome coronavirus 2 (SARSCoV-2) disease 2019 (COVID-19) raised concern for the outcomes in people with different rheumatic diseases and management of these patients. There was an anxiety that biologic therapies, especially anti-B-cell depletion strategies, could lead to more severe disease course and lack of protective antibodies formation. 